\title{
Expression of AQP3 and AQP5 as a prognostic marker in triple-negative breast cancer
}

\author{
ZHENGCAI ZHU $^{1,2^{*}}$, LIANGHE JIAO ${ }^{2 *},{\text { TAO } \mathrm{LI}^{2 *}, \mathrm{HONGGANG} \mathrm{WANG}^{2}, \text { WEI WEI }^{3} \text { and HAIXIN QIAN }}^{1}$ \\ ${ }^{1}$ Department of General Surgery, The First Affiliated Hospital of Soochow University, Suzhou, Jiangsu 215000; \\ ${ }^{2}$ Department of General Surgery, Taizhou People's Hospital, Taizhou, Jiangsu 215300; ${ }^{3}$ Department of General Surgery, \\ Nanjing Medical University Affiliated Cancer Hospital, Nanjing, Jiangsu 210029, P.R. China
}

Received October 31, 2017; Accepted April 30, 2018

DOI: $10.3892 / \mathrm{ol} .2018 .8955$

\begin{abstract}
Triple-negative breast cancer (TNBC) is a common type of breast malignancy with high a propensity for metastasis and locoregional recurrence. The aim of the present study was to investigate the expression of aquaporin (AQP) 3 and AQP5, analyze their association with clinicopathological parameters and explore their clinical significance in tissue samples from patients with TNBC. Immunohistochemistry was performed to detect the expression patterns of AQP3 and AQP5 in 96 patients with TNBC who underwent surgery between 2007 and 2012. AQP3 and AQP5 were expressed primarily in the membrane and cytoplasm of tumor cells within TNBC tissues. AQP3 and AQP5 expression was notably stronger in carcinoma tissue compared with adjacent normal tissue. Overexpression of AQP3 and AQP5 was significantly associated with tumor size, lymph node status and local relapse/distant metastasis. In addition, aberrant overexpression of AQP5 was observed more frequently in TNBC tissues with higher Ki-67 expression than in those with lower Ki-67 expression. In univariate analysis, patients with TNBC with high AQP3 and AQP5 expression demonstrated poorer 5-year disease-free survival and overall survival compared with patients with low AQP3 and AQP5 expression. In multivariate analysis, the combined expression of AQP3 and AQP5 was an independent prognostic marker in patients with TNBC. The results of the present study suggest that the overexpression of AQP3 and AQP5 may serve as a novel therapeutic marker in patients with TNBC.
\end{abstract}

Correspondence to: Dr Haixin Qian, Department of General Surgery, The First Affiliated Hospital of Soochow University, 188 Shizi Road, Suzhou, Jiangsu 215000, P.R. China

E-mail: zhuzhengcai8237@163.com

${ }^{*}$ Contributed equally

Key words: triple-negative breast cancer, aquaporin 3, aquaporin 5, prognosis

\section{Introduction}

Breast cancer is the most common malignancy affecting women's health, with increasing incidence worldwide. About 1 in 8 women will be diagnosed with breast cancer in their lifetimes; moreover, 1 in 5 cases of breast cancer is the triple-negative subtype, that is, negative for estrogen receptor, progesterone receptor, and human epidermal growth factor receptor 2 (HER2) (1). Triple-negative breast cancer (TNBC) tends to be particularly aggressive, with a higher propensity for metastasis and locoregional recurrence compared with other subtypes (2). Although several prognostic criteria and markers have already been introduced to assist management after curative surgery for TNBC (3), identifying novel molecular markers in order to discriminate individual variability and predict survival and thus provide individualized treatment is necessary.

Aquaporins (AQPs), a family of small (30 kDa/monomer) water channel proteins that are integral membrane proteins, play a crucial role in water homeostasis by regulating cellular water transport (4). Thus far, 13 AQPs (AQP0-AQP12) have been identified, and their expression is widely distributed in various tissues throughout the body. Among them, AQP0, AQP1, AQP2, AQP4, AQP5, AQP6, and AQP8 are primarily involved in water transport, whereas AQP3, AQP7, AQP9, and AQP10 are also involved in the transport of other small solutes, such as glycerol and urea (5). AQP11 and AQP12, also named 'superaquaporins' or 'subcellular aquaporins,' are located intracellularly with no clearly established selectivity (6). Accumulating studies have revealed that AQPs are involved in many physiological functions, such as urine concentration, exocrine gland secretion, brain swelling, neural signal transduction, skin moisturization, and fat metabolism (7-10).

Recently, some studies have demonstrated certain AQP subtypes as novel targets for antitumor therapy because of their involvement in carcinogenesis, tumor progression, and invasion $(11,12)$. AQP3 and AQP5 are highly expressed in stomach, tongue, liver, pancreatic, colorectal, lung, and cervical cancers (13-16). In previous studies, prominent AQP3 and AQP5 expression was also observed in breast cancer tissues (17). However, to date, there has been no investigation of the clinicopathological relevance of AQP3 and AQP5 expression in TNBC. Accordingly, the aim of this study was 
to investigate the expression patterns of AQP3 and AQP5 and to evaluate their relationships with clinicopathological characteristics and prognosis in TNBC patients.

\section{Materials and methods}

Patient and tissue samples. The present study was approved by the Research Ethics Committees of Jiangsu Taizhou People's Hospital and Soochow University, Suzhou, China. Written informed consent was obtained from all patients. All specimens were handled anonymously according to the local ethical and legal standards.

A total of ninety-six tumor samples and matched adjacent normal samples were obtained from 96 women who underwent surgery for TNBC at Jiangsu Taizhou People's Hospital, China between 2007 and 2012. Patients with ductal carcinoma in situ, lobular carcinoma, or those who underwent any type of neoadjuvant treatment prior to surgery were not enrolled in the present study. The average patient age was $48.86 \pm 10.32$ years. All patients were classified according to the pathological tumor/node/metastasis (TNM) system based on the seventh edition of America Joint Committee on Cancer. Clinicopathological characteristics, including age, menopausal status, operation method, histological grade, tumor size, nodal status, and local relapse/distant metastasis, are summarized in Table I.

The postoperative evaluation of all TNBC patients was followed up every 3 months for the first 2 years, then at least every 6 months thereafter. Follow-up was completed on June 30, 2017. The median follow-up time was 39 months (range 5-60 months). We excluded any patient with concurrent non-breast malignant tumors or non-tumor related death. Hence, any death reported in the cases enrolled in this study is attributed to the original TNBC tumor, its recurrence or distant metastasis. At each follow-up visit, a complete medical history was taken and clinical examinations were performed. Tests for tumor markers such as carcinoembryonic antigen (CEA), cancer antigen 125 (CA125), and cancer antigen 153 (CA153), mammogram, and ultrasound were performed every 3-12 months. CT, MRI, or FDG-PET/CT was performed selectively in case of any abnormalities during the examination. Relapse or metastasis was confirmed by biopsy where possible. Disease-free survival (DFS) and overall survival (OS) were defined as the time from the date of surgery to the date of recurrence and the date of the last follow-up or death, respectively.

Immunohistochemistry analysis. Immunohistochemistry was performed on formalin-fixed, paraffin-embedded, 4- $\mu \mathrm{m}$ tissue sections using the EnVision complex method. Briefly, the Ethylene Diamine Tetraacetic Acid (EDTA) heat repair method was performed for $20 \mathrm{~min}$ for antigen retrieval, and sections were placed in an endogenous peroxidase blocker for $15 \mathrm{~min}$ at room temperature to quench endogenous peroxidase. The slides were washed thrice (1 min each) with phosphate-buffered saline (PBS) and incubated with the primary antibodies against AQP3 (rabbit polyclonal antibody, cat no.: ab-125219; Abcam, Cambridge, MA, USA) and AQP5 (rabbit monoclonal antibody, cat no.: ab-92320; Abcam) at a 1:500 dilution overnight at $4^{\circ} \mathrm{C}$. After several washes, the sections were incubated in the EnVision ${ }^{\text {TII }}+/$ HRP rabbit working solution (Dako; Agilent
Technologies, Inc., Santa Clara, CA, USA) for $30 \mathrm{~min}$ and then stained with diaminobenzidine. The slides were washed with distilled water and counterstained with hematoxylin. After thorough washes, each chip was dehydrated and then sealed with a neutral gum. In each immunohistochemistry run, PBS was used instead of the primary antibody as a negative control, and an internal control was used for each slide.

Immunolabeled slides were scored by two independent expert pathologists blinded to the clinicopathological data and clinical prognosis. Owing to the homogeneousness of the immunostaining of the target proteins, tumor specimens were scored in a semiquantitative manner on the basis of a well-established immunoreactivity scoring system (IRS) (18). The staining intensity score (IS) was stratified as follows: 0 (no staining), 1 (faint), 2 (moderate), and 3 (strong). The percentage of positive cells (PC) was scored as follows: $0(0 \%)$, 1 (1-10\%), 2 (11-50\%), $3(51-80 \%)$, and 4 (>80\%). The final IRS was obtained for each case by multiplying the IS and PC. Expression levels of target protein were further analyzed by stratifying IRS values as low ( $\leq$ the average value) and high ( $>$ the average value).

Statistical analysis. SPSS v21.0 software (SPSS, Inc., Chicago, IL, USA) was used for statistical analysis. Results are expressed as mean and standard deviation. Student's t-test was used to compare the expression levels of AQP3 and AQP5 between carcinoma tissues and adjacent normal tissues. Categorical variables were compared using the chi-square test. The correlation between the expression levels of AQP3, AQP5, and Ki-67 was analyzed using the Spearman's rank correlation coefficient. The survival curves were constructed based on the Kaplan-Meier method. Cox proportional hazards regression models were performed for the univariate and multivariate analysis. $\mathrm{P}<0.05$ was considered to indicate a statistically significant difference.

\section{Results}

Expression and localization of AQP3 and AQP5 in TNBC and adjacent normal tissue. $\mathrm{AQP} 3$ and $\mathrm{AQP} 5$ were expressed mainly in the membrane and cytoplasm of tumor cells in TNBC tissues. AQP3 (IRS: $6.31 \pm 1.24$ vs. $2.87 \pm 0.58, \mathrm{P}<0.001$ ) and AQP5 (IRS: $5.95 \pm 1.36$ vs. $2.96 \pm 0.43, \mathrm{P}<0.001$ ) expression was remarkably stronger in the carcinoma tissues than in the adjacent normal tissues (Fig. 1). Moreover, in some of the TNBC tissues, AQP5 was more prominent on the invasive front, and AQP5 staining was decreased in areas adjacent to necrosis (Fig. 1C). In adjacent normal tissues, however, there was weak immunostaining of AQP3 and AQP5 in the periductal or intralobular stroma, and little in the endothelial cells of the capillary, small veins, and peripheral nerve fibers (Fig. 1B and D).

Relationship between clinicopathological features and AQP3/AQP5 expression in TNBC patients. To evaluate whether AQP3 and AQP5 expression was associated with clinicopathological features of TNBC patients, we investigated the association of AQP3 and AQP5 expression with age, menopausal status, surgical method, histological grade, tumor size, nodal status, local relapse/distant metastasis and Ki-67 
Table I. Association of AQP3 and AQP5 expression with established clinicopathological parameters in 96 patients with TNBC.

\begin{tabular}{|c|c|c|c|c|c|c|c|}
\hline \multirow[b]{2}{*}{ Factor } & \multirow[b]{2}{*}{ No. } & \multicolumn{2}{|c|}{ AQP3 expression n (\%) } & \multirow[b]{2}{*}{ P-value } & \multicolumn{2}{|c|}{ AQP5 expression n (\%) } & \multirow[b]{2}{*}{ P-value } \\
\hline & & Low & High & & Low & High & \\
\hline \multicolumn{8}{|l|}{ Age } \\
\hline$\leq 50$ years & 51 & $21(41.2)$ & $30(58.8)$ & \multirow[t]{2}{*}{0.734} & $24(47.1)$ & $27(52.9)$ & \multirow[t]{2}{*}{0.172} \\
\hline$>50$ years & 45 & $17(37.8)$ & $28(62.2)$ & & $15(33.3)$ & $30(66.7)$ & \\
\hline \multicolumn{8}{|l|}{ Menopausal status } \\
\hline Pre-menopause & 58 & $23(39.7)$ & $35(60.3)$ & \multirow[t]{2}{*}{0.986} & $26(44.8)$ & $32(55.2)$ & \multirow[t]{2}{*}{0.300} \\
\hline Post-menopause & 38 & $15(39.5)$ & $23(60.5)$ & & $13(34.2)$ & $25(65.8)$ & \\
\hline \multicolumn{8}{|l|}{ Surgery } \\
\hline Mastectomy & 67 & $25(37.3)$ & $42(62.7)$ & \multirow[t]{2}{*}{0.489} & $27(40.3)$ & $40(59.7)$ & \multirow[t]{2}{*}{0.921} \\
\hline Breast conserving & 29 & $13(44.8)$ & $16(55.2)$ & & $12(41.4)$ & $17(58.6)$ & \\
\hline \multicolumn{8}{|l|}{ Histological grade } \\
\hline I & 16 & $5(31.3)$ & $11(68.8)$ & \multirow[t]{3}{*}{0.321} & $7(43.8)$ & $9(56.3)$ & \multirow[t]{3}{*}{0.287} \\
\hline II & 44 & $21(47.7)$ & $23(52.3)$ & & $21(47.7)$ & $23(52.3)$ & \\
\hline III & 36 & $12(33.3)$ & $24(66.7)$ & & $11(30.6)$ & $25(69.4)$ & \\
\hline \multicolumn{8}{|l|}{ Tumor size } \\
\hline $\mathrm{T} 1$ & 36 & $20(55.6)$ & $16(44.4)$ & \multirow[t]{3}{*}{0.035} & $22(61.1)$ & $14(38.9)$ & \multirow[t]{3}{*}{0.001} \\
\hline $\mathrm{T} 2$ & 46 & $15(32.6)$ & $31(67.4)$ & & $16(34.8)$ & $30(65.2)$ & \\
\hline T3 T4 & 14 & $3(21.4)$ & $11(78.6)$ & & $1(7.1)$ & $13(92.9)$ & \\
\hline \multicolumn{8}{|l|}{ Nodal status } \\
\hline Negative & 59 & $28(47.5)$ & $31(52.5)$ & \multirow[t]{2}{*}{0.046} & $30(50.8)$ & $29(49.2)$ & \multirow[t]{2}{*}{0.010} \\
\hline Positive & 37 & $10(27.0)$ & $27(73.0)$ & & $9(24.3)$ & $28(75.7)$ & \\
\hline \multicolumn{8}{|l|}{ Distant metastasis } \\
\hline M0 & 79 & $36(45.6)$ & $43(54.4)$ & \multirow[t]{2}{*}{0.021} & $38(48.1)$ & $41(51.9)$ & \multirow[t]{2}{*}{0.003} \\
\hline M1 & 17 & $2(11.8)$ & $15(88.2)$ & & $1(5.9)$ & $16(94.1)$ & \\
\hline \multicolumn{8}{|l|}{ Ki-67 level } \\
\hline Low expression & 18 & $8(44.4)$ & $10(55.6)$ & \multirow[t]{2}{*}{0.640} & $12(66.7)$ & $6(33.3)$ & \multirow[t]{2}{*}{0.013} \\
\hline High expression & 78 & $30(38.5)$ & $48(61.5)$ & & $27(34.6)$ & $51(65.4)$ & \\
\hline
\end{tabular}

TNBC, triple-negative breast cancer; AQP, aquaporin.

Table II. Correlation of AQP3, AQP5 and Ki-67 in 96 patients with TNBC.

\begin{tabular}{lcrccc}
\hline & & \multicolumn{2}{c}{ Ki-67 } & & \\
\cline { 3 - 4 } & Expression & Low (n) & High (n) & r-value & P-value \\
\hline \multirow{2}{*}{ AQP3 } & Low & 8 & 30 & 0.048 & 0.644 \\
& High & 10 & 48 & & \\
AQP5 & Low & 12 & 27 & 0.255 & 0.012 \\
& High & 6 & 51 & &
\end{tabular}

TNBC, Triple negative breast cancer; AQP, aquaporin.

expression (Table I). The average IRS values of AQP3 and AQP5 expression in TNBC tissues were 6.31 and 5.95, respectively. TNBC patients with AQP3 or AQP5 expression less than or equal to the average value were assigned to the AQP3-low or AQP5-low expression group, and those with expression above the average level were assigned to the AQP3-high or AQP5-high expression group. As shown in Table I, combined AQP3 and AQP5 overexpression was significantly associated with tumor size $(\mathrm{P}=0.035$ and 0.001 , respectively), nodal status ( $\mathrm{P}=0.046$ and 0.01 , respectively), and local relapse/distant metastasis $(\mathrm{P}=0.021$ and 0.003 , respectively). There was no significant difference in age, menopausal status, surgical method, or histological grade between the groups. In addition, aberrant overexpression of AQP5 was observed more frequently in TNBC tissues with high $\mathrm{Ki}-67$ expression than in those with low Ki-67 expression $(\mathrm{P}=0.013)$. As analyzed by the Spearman's rank correlation analysis, AQP5 expression was closely associated with $\mathrm{ki}-67$ expression $(\mathrm{r}=0.255$, $\mathrm{P}=0.012$; Table II). A similar correlation was not found for AQP3 ( $r=0.048, \mathrm{P}=0.064$; Table II).

Univariate analyses were performed to evaluate the effect of co-expression of AQP3 and AQP5 and other clinicopathological parameters on TNBC prognosis. Five-year DFS and OS of TNBC patients in our study were significantly associated 
A

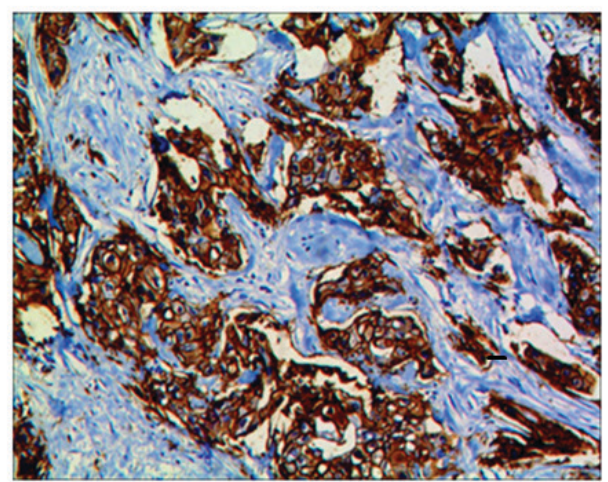

C

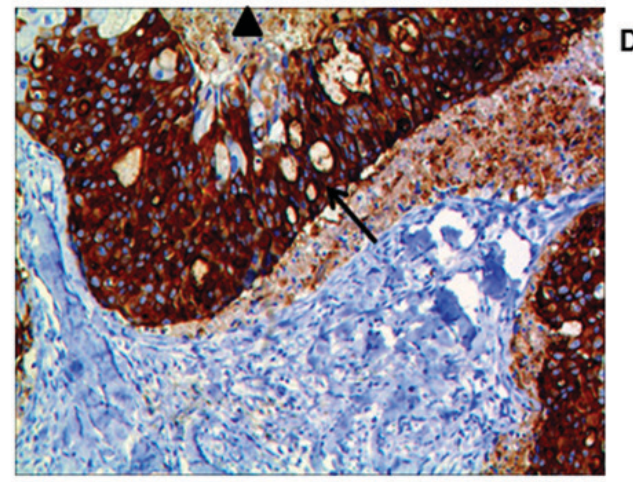

B
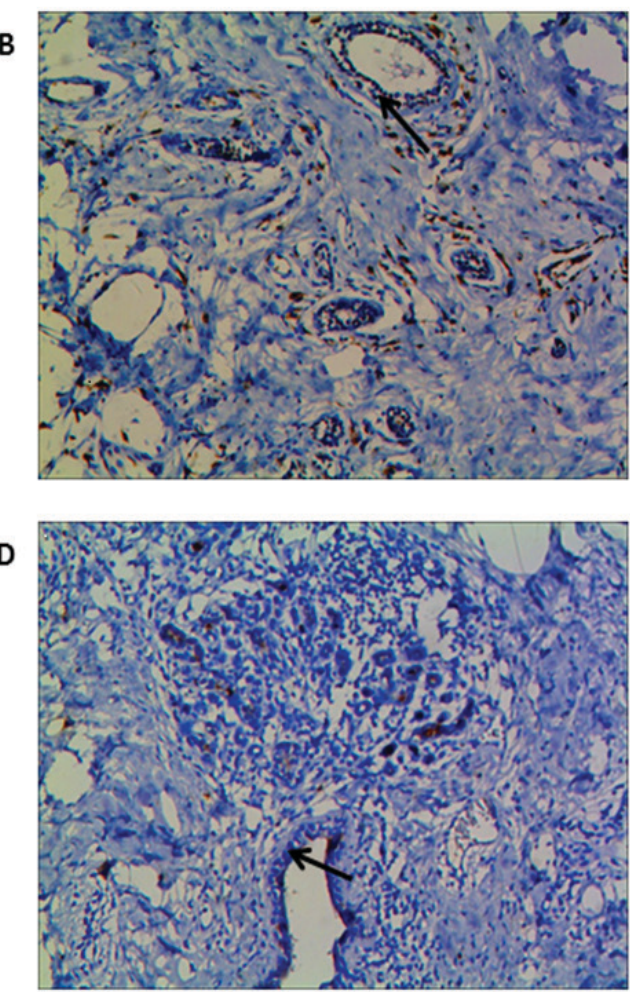

Figure 1. Immunohistochemical staining for AQP3 and AQP5 proteins in TNBC tissues and adjacent normal tissues. AQP3 (A) and AQP5 (C) were expressed mainly in the membrane and cytoplasm of tumor cells in TNBC tissues, and their expression was stronger in the carcinoma tissues than in the adjacent normal tissues. Furthermore, immunostaining of AQP5 was more prominent on the invasive front of the tumor (C, arrow $\uparrow)$, and decreased near necrotic areas $(\mathrm{C}$, triangle $\mathbf{\Lambda})$. (B and D) In adjacent normal tissues, however, there was weak immunostaining of AQP3 and AQP5 in the periductal or intralobular stroma, and little in the endothelial cells of the capillary, small veins, and peripheral nerve fibers. Magnification, x200. AQP, aquaporin; TNBC, triple-negative breast cancer.

with increased expression of both AQP3 and AQP5 $(\mathrm{P}=0.015$ and $\mathrm{P}=0.028$, respectively), tumor size $(\mathrm{P}=0.036$ and 0.041 , respectively), nodal status $(\mathrm{P}=0.026$ and 0.018 , respectively), local relapse/distant metastasis $(\mathrm{P}=0.018$ and 0.021 , respectively), and $\mathrm{Ki}-67$ expression ( $\mathrm{P}=0.032$ and 0.043 , respectively) (Table III).The significant parameters in univariate analysis were further evaluated in multivariate analysis. We found that nodal status $(\mathrm{P}=0.026$ and 0.038 , respectively), local relapse/distant metastasis $(\mathrm{P}=0.032$ and 0.039 , respectively), and increased expression of both AQP3 and AQP5 ( $\mathrm{P}=0.014$ and 0.025 , respectively) were independent poor prognostic factors for OS and DFS in TNBC patients (Table IV).

\section{Discussion}

TNBC is associated with a younger age, a higher mitotic index and more advanced stage at diagnosis. Because neither endocrine nor targeted therapies are effective in the treatment of TNBC, its prognosis is poor compared with other breast cancer subtypes (19). Therefore, determining markers that can both serve as prognosticators and potential therapeutic targets are urgently required to advance effective treatment approaches. In the current study, we investigated the expression patterns of AQP3 and AQP5 in 96 TNBC patients through immunohistochemistry. Overexpression of AQP3 and AQP5 was observed in 60.41 and $59.37 \%$ of TNBC tissues, respectively. Both AQP3 and AQP5 proteins were expressed mainly in the membrane and cytoplasm of tumor cells. Furthermore, immunostaining of AQP5 was more prominent on the invasive front of the tumor, and decreased near necrotic areas. Besides, combined AQP3 and AQP5 overexpression was significantly associated with tumor size, nodal status, and local relapse/distant metastasis in TNBC patients. Aberrant overexpression of AQP5 protein was observed more frequently in patients with higher Ki-67 than in those with lower Ki-67. Moreover, pairwise comparisons showed that the patients with AQP3-high/AQP5-high expression had the poorest DFS and OS. In multivariate analysis, high expression of AQP3 and AQP5 was found to be an independent prognostic factor of relapse and decreased survival for TNBC. To the best of our knowledge, there are no reports on the co-expression of AQP3 and AQP5 on TNBC.

AQPs are a family of water-transporting integral membrane proteins. However, increasing evidence shows that AQPs play important roles in tumorigenesis, tumor progression, invasion, and metastasis (20). AQP3 and AQP5, as subtypes of the AQP family, are overexpressed in a variety of tumor types, suggesting an important role in tumorigenesis. We have reported on the high expression of AQP3 and AQP5 in gastric cancer and their important role in the migration and proliferation of gastric cancer cells, suggesting that AQP3 and AQP5 may be potentially important determinants of tumor growth and metastasis $(21,22)$. In lung cancer, overexpression of AQP3 is associated with tumor pathological grade and clinical stage (23). In a mouse model, AQP3 knockdown inhibits tumor growth and decreases angiogenesis in human non-small cell lung cancer xenografts (24). In human breast cancer, Shi et al found that AQP1 and AQP3-5 exhibited differential expression 

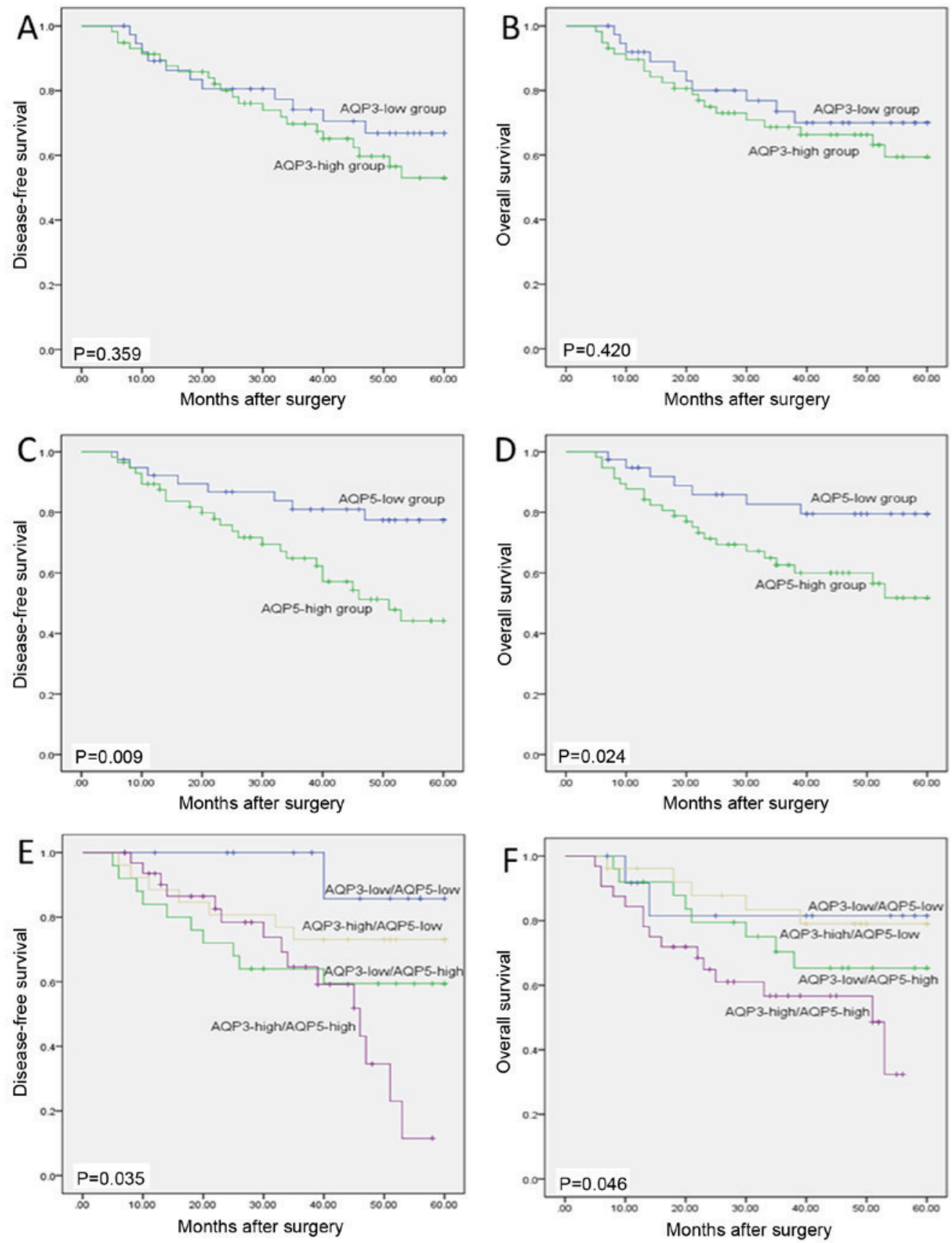

Figure 2. Kaplan-Meier survival curves for AQP3 expression, AQP5 expression and combined expression of AQP3 and AQP5 in TNBC tissues. 5-year DFS (C) and OS (D) in the group with high AQP5 expression were poorer than those in the group with low AQP5 expression ( $\mathrm{P}=0.009, \mathrm{P}=0.024$, respectively). However, no significant difference in DFS (A) or OS (B) was found in the high and low AQP3 expression subgroups ( $\mathrm{P}=0.359$, $\mathrm{P}=0.420$, respectively). Besides, pairwise comparison showed that patients with AQP3-high/AQP5-high expression had the poorest DFS (E) and OS (F) among all the groups (P=0.035, $\mathrm{P}=0.046$, respectively). AQP, aquaporin; TNBC, triple-negative breast cancer; DFS, disease-free survival; OS, overall survival.

between breast cancer and normal breast tissues, suggesting that some subtypes of the AQP family play a key role in human breast carcinogenesis (25). Kang et al (26), demonstrated that, in patients with early HER2-positive breast cancer, AQP3 expression was significantly related to survival and was an independent prognostic marker of DFS. Furthermore, the common chemotherapy drug cisplatin induces ovarian tumor cell death by inhibiting AQP5 expression and the NF- $\kappa \mathrm{B}$ pathway, supporting AQP5 as a potential target for therapy in ovarian cancer (27). Similarly, expression of both AQP3 and AQP5 are increased in squamous cell carcinoma. Treatment with the AQP inhibitor, $\mathrm{CuSO}_{4}$, or AQP5-specific siRNA shows inhibition of cell growth in squamous cell carcinoma cell lines via the inhibition of integrins and the mitogen-activated protein kinase pathway (28).

Little is known about the expression and role of AQP3 and AQP5 in TNBC. The current study indicates that AQP3 and AQP5 overexpression were positively correlated with Ki-67 expression, tumor size, lymph node metastasis, and local relapse/distant metastasis. The biologic marker Ki-67 has been identified as an important prognostic and predictive marker in breast cancer (29). Moreover, Guo et al (14) demonstrated 
Table III. Univariate analysis of different prognostic factors in 96 patients with TNBC.

\begin{tabular}{|c|c|c|c|c|}
\hline \multirow[b]{2}{*}{ Factor } & \multicolumn{2}{|c|}{ Overall survival } & \multicolumn{2}{|c|}{ Disease-free survival } \\
\hline & HR $(95 \% \mathrm{CI})$ & P-value & HR (95\% CI) & P-value \\
\hline Age & $1.025(0.948-1.108)$ & 0.534 & $1.049(0.857-1.284)$ & 0.643 \\
\hline Menopausal & $1.125(0.820-1.543)$ & 0.465 & $1.624(0.541-4.872)$ & 0.387 \\
\hline Surgery & $1.434(0.811-2.535)$ & 0.215 & $1.826(1.566-5.896)$ & 0.314 \\
\hline Histological grade & $2.231(0.390-12.755)$ & 0.367 & $1.526(0.557-4.180)$ & 0.411 \\
\hline Tumor size & $1.845(1.041-3.271)$ & 0.036 & $1.485(1.016-2.170)$ & 0.041 \\
\hline Nodal status & $2.044(1.089-3.836)$ & 0.026 & $2.851(1.197-6.792)$ & 0.018 \\
\hline Distant metastasis & $4.426(1.291-15179)$ & 0.018 & $3.895(1.228-12.359)$ & 0.021 \\
\hline Ki-67 & $1.174(1.014-1.359)$ & 0.032 & $1.252(1.007-1.556)$ & 0.043 \\
\hline AQP3/AQP5 expression & $4.356(1.331-14.258)$ & 0.015 & $3.892(1.158-13.080)$ & 0.028 \\
\hline
\end{tabular}

For AQP3/AQP5 expression, AQP3 and AQP5 expression levels above 6.31 and 5.95 were considered, respectively; TNBC, Triple negative breast cancer; AQP, aquaporin; HR, hazard's ratio; CI, confidence interval.

Table IV. Multivariate analysis of different prognostic factors in 96 patients with TNBC.

\begin{tabular}{|c|c|c|c|c|}
\hline \multirow[b]{2}{*}{ Factors } & \multicolumn{2}{|c|}{ Overall survival } & \multicolumn{2}{|c|}{ Disease-free survival } \\
\hline & HR $(95 \%$ CI $)$ & P-value & HR $(95 \% \mathrm{CI})$ & $\mathrm{P}$-value \\
\hline Tumor size & $1.151(0.979-1.354)$ & 0.089 & $1.088(0.977-1.212)$ & 0.126 \\
\hline Nodal status & $1.847(1.076-3.170)$ & 0.026 & $1.775(1.032-3.052)$ & 0.038 \\
\hline Distant metastasis & 3.245 (1.107-9.516) & 0.032 & $4.122(1.074-15.818)$ & 0.039 \\
\hline Ki-67 & $1.266(0.952-1.684)$ & 0.105 & $1.528(0.812-2.877)$ & 0.189 \\
\hline AQP3/AQP5 expression & $5.324(1.403-20.207)$ & 0.014 & $4.248(1.199-15.049)$ & 0.025 \\
\hline
\end{tabular}

For AQP3/AQP5 expression, AQP3 and AQP5 expression levels above 6.31 and 5.95 were considered, respectively; TNBC, Triple negative breast cancer; HR, hazard's ratio; CI, confidence interval; AQP, aquaporin.

that the overexpression of AQP3 in combination with upregulation of AQP5 was an unfavorable prognostic factor in hepatocellular carcinoma. Therefore, combined expression of AQP3 and AQP5 may be a potential promising marker in TNBC. Excitingly, AQPs have been targeted in the clinical treatment of some diseases, such as nephrogenic diabetes insipidus (AQP2), and neuromyelitis optica, an autoimmune demyelinating disease (AQP4) $(30,31)$, identifying AQP3 and AQP5 as novel targets for anti-cancer treatment still need further investigation.

Though our study generated some important findings, a limitation due to the relatively short follow-up and small sample size should be acknowledged. Only immunohistochemistry was performed in present study, and mRNA and protein expression of AQP3 and AQP5 were not assessed in cancer tissues. Additionally, the molecular mechanisms for this aberrant expression, as well as the roles of AQP3 and AQP5 in TNBC patients, have not been fully elucidated. Further studies are needed to more fully understand their molecular function in TNBC.

In summary, the present study demonstrates for the first time that increased co-expression of AQP3 and AQP5 may be associated with tumor clinicopathological characteristics and could serve as an independent prognostic factor in TNBC patients. Combined expression of the two proteins may be a potential promising marker in patients with TNBC and could be a novel target for anti-cancer treatment.

\section{Acknowledgements}

The authors would like to thank Professor Lizong Shen (Division of Gastrointestinal Surgery, Department of General Surgery, First Affiliated Hospital, Nanjing Medical University) for his helpful suggestions.

\section{Funding}

The present study was supported by the National Natural Science Foundation of China (grant no. 81600434).

\section{Availability of data and materials}

All data generated or analyzed during this study are included in this published article. 


\section{Author's contributions}

ZCZ, LHJ and LT performed the surgery and drafted the manuscript. ZCZ and HGW participated in the design of the study and performed the histological examination. WW performed the statistical analysis. HXQ made substantial contributions to the manuscript including conception and design, acquisition of data, analysis and interpretation of data and contributed to the supervision and revision of the manuscript. All authors read and approved the final manuscript.

\section{Ethics approval and consent to participate}

All study participants provided informed consent and the study design was approved by the Research Ethics Committees of Jiangsu Taizhou People's Hospital and Soochow University (Suzhou, China).

\section{Consent for publication}

Written informed consent for publication of their clinical details and images was obtained from all participants in the present study.

\section{Competing interests}

The authors declare that they have no competing interests.

\section{References}

1. Chen VE, Gillespie EF, Zakeri K, Murphy JD, Yashar CM, Lu S and Einck JP: Pathologic response after neoadjuvant chemotherapy predicts locoregional control in patients with triple negative breast cancer. Adv Radiat Oncol 2: 105-109, 2017.

2. Santana-Davila R and Perez EA: Treatment options for patients with triple-negative breast cancer. J Hematol Oncol 3: 42, 2010.

3. Verkman AS, Anderson MO and Papadopoulos MC: Aquaporins: Important but elusive drug targets. Nat Rev Drug Discov 13 259-277, 2014.

4. Lee SJ, Chae YS, Kim JG, Kim WW, Jung JH, Park HY, Jeong JY, Park JY, Jung HJ and Kwon TH: AQP5 expression predicts survival in patients with early breast cancer. Ann Surg Oncol 21: 375-383, 2014

5. Verkman AS: Aquaporins in clinical medicine. Annu Rev Med 63: 303-316, 2012.

6. Ishibashi K, Tanaka Y and Morishita Y: The role of mammalian superaquaporins inside the cell. Biochim Biophys Acta 1840: $1507-1512,2014$

7. Li Y, Wang W, Jiang T and Yang B: Aquaporins in urinary system. Adv Exp Med Biol 969: 131-148, 2017.

8. Liu S, Zhang S, Jiang H, Yang Y and Jiang Y: Co-expression of AQP3 and AQP5 in esophageal squamous cell carcinoma correlates with aggressive tumor progression and poor prognosis. Med Oncol 30: 636, 2013.

9. Filippidis AS, Carozza RB and Rekate HL: Aquaporins in brain edema and neuropathological conditions. Int J Mol Sci 18: pii: E55, 2016.

10. Ikarashi N, Kon R, Kaneko M, Mizukami N, Kusunoki Y and Sugiyama K: Relationship between aging-related skin dryness and aquaporins. Int J Mol Sci 18: pii: E1559, 2017.

11. Esteva-Font C, Jin BJ and Verkman AS: Aquaporin-1 gene deletion reduces breast tumor growth and lung metastasis in tumor-producing MMTV-PyVT mice. FASEB J 28: 1446-1453, 2014.
12. Kang BW, Kim JG, Lee SJ, Chae YS, Jeong JY, Yoon GS, Park SY, Kim HJ, Park JS, Choi GS and Jeong JY: Expression of aquaporin-1, aquaporin-3, and aquaporin-5 correlates with nodal metastasis in colon cancer. Oncology 88: 369-376, 2015.

13. Chen J, Wang T, Zhou YC, Gao F, Zhang ZH, Xu H, Wang SL and ShenLZ: Aquaporin 3 promotes epithelial-mesenchymaltransition in gastric cancer. J Exp Clin Cancer Res 33: 38, 2014.

14. Guo X, Sun T, Yang M, Li Z, Li Z and Gao Y: Prognostic value of combined aquaporin 3 and aquaporin 5 overexpression in hepatocellular carcinoma. Biomed Res Int 2013: 206525, 2013.

15. Direito I, Paulino J, Vigia E, Brito MA and Soveral G: Differential expression of aquaporin-3 and aquaporin-5 in pancreatic ductal adenocarcinoma. J Surg Oncol 115: 980-996, 2017.

16. Ribatti D, Ranieri G, Annese T and Nico B: Aquaporins in cancer. Biochimica et Biophysica Acta 1840: 1550-1553, 2014.

17. Wang J, Feng L, Zhu Z, Zheng M, Wang D, Chen Z and Sun H: Aquaporins as diagnostic and therapeutic targets in cancer: How far we are? J Transl Med 13: 96, 2015.

18. Gatto F, Feelders RA, van der Pas R, Kros JM, Waaijers M, Sprij-Mooij D, Neggers SJ, van der Lelij AJ, Minuto F, Lamberts SW, et al: Immunoreactivity score using an anti-sst2A receptor monoclonal antibody strongly predicts the biochemical response to adjuvant treatment with somatostatin analogs in acromegaly. J Clin Endocrinol Metab 98: E66-E71, 2013.

19. Rampurwala M, Wisinski KB and Regan RO: Role of the androgen receptor in triple-negative breast cancer. Clin Adv Hematol Oncol 14: 186-193, 2016.

20. Satooka $\mathrm{H}$ and Hara-Chikuma M: Aquaporin-3 controls breast cancer cell migration by regulating hydrogen peroxide transport and its downstream cell signaling. Mol Cell Biol 36: 1206-1218, 2016.

21. Shen L, Zhu Z, Huang Y, Shu Y, Sun M, Xu H, Zhang G, Guo R, Wei $\mathrm{W}$ and $\mathrm{Wu} \mathrm{W}$ : Expression profile of multiple aquaporins in human gastric carcinoma and its clinical significance. Biomed Pharmacother 64: 313-318, 2010.

22. Huang Y, Zhu Z, Sun M, Wang J, Guo R, Shen L and Wu W: Critical role of aquaporin-3 in the human epidermal growth factor-induced migration and proliferation in the human gastric adenocarcinoma cells. Cancer Biol Ther 9: 1000-1007, 2010.

23. Papadopoulos MC and Saadoun S: Key roles of aquaporins in tumor biology. Biochim Biophys Acta 1848: 2576-2583, 2015.

24. Xia H, Ma YF, Yu CH, Li YJ, Tang J, Li JB, Zhao YN and Liu Y: Aquaporin 3 knockdown suppresses tumour growth and angiogenesis in experimental non-small cell lung cancer. Exp Physiol 99: 974-984, 2014.

25. Shi Z, Zhang T, Luo L, Zhao H, Cheng J, Xiang J and Zhao C: Aquaporins in human breast cancer: Identification and involvement in carcinogenesis of breast cancer. J Surg Oncol 106: 267-272, 2012.

26. Kang S, Chae YS, Lee SJ, Kang BW, Kim JG, Kim WW, Jung JH, Park HY, Jeong JH, Jeong JY and Park JY: Aquaporin 3 expression predicts survival in patients with HER2-positive early breast cancer. Anticancer Res 35: 2775-2782, 2015.

27. Yang J, Yan C, Zheng W and Chen X: Proliferation inhibition of cisplatin and aquaporin 5 expression in human ovarian cancer cell CAOV3. Arch Gynecol Obstet 285: 239-245, 2012.

28. Ishimoto S, Wada K, Usami Y, Tanaka N, Aikawa T, Okura M, Nakajima A, Kogo M and Kamisaki Y: Differential expression of aquaporin 5 and aquaporin 3 in squamous cell carcinoma and adenoid cystic carcinoma. Int J Oncol 41: 67-75, 2012.

29. Schlotter CM, Tietze L, Vogt U, Heinsen CV and Hahn A: Ki67 and lymphocytes in the pretherapeutic core biopsy of primary invasive breast cancer: Positive markers of therapy response prediction and superior survival. Horm Mol Biol Clin Investig 32: pii: /j/hmbci, 2017.

30. Kowarik MC, Soltys J and Bennett JL: The treatment of neuromyelitis optica. J Neuroophthalmol 34: 70-82, 2014.

31. Soveral $\mathrm{G}$ and Casini A: Aquaporin modulators: A patent review (2010-2015). Expert Opin Ther Pat 27: 49-62, 2017. 